\title{
Temporización inmediata de implantes unitarios en la maxila posterior
}

\author{
Immediate temporization of posterior maxilla single tooth implant
}

\author{
Leighton $\mathrm{Y}^{1}$, Carvajal $\mathrm{JC}^{2}$, Wolnitzky $\mathrm{A}^{3}$, Silva $\mathrm{R}^{4}$, Von Marttens $\mathrm{A}^{5}$
}

\begin{abstract}
RESUMEN
Propósito: El presente artículo es un estudio prospectivo de las tasas de éxito implantaria y de las complicaciones mecánicas y biológicas en el tratamiento rehabilitador implanto-asistido del sector posterior de la maxila, mediante provisionalización inmediata. Material y Método: El estudio clínico observó a 21 sujetos desdentados parciales unitarios del sector posterior maxilar, con reborde cicatrizado y antagonista dentario natural, por un período de 6 meses, en los que se insertaron implantes cónicos roscados de conexión interna, con tratamiento de superficie, temporizados inmediatamente después de insertados en el hueso maxilar, mediante la confección de una corona de resina acrílica sobre un pilar temporal de titanio atornillada directa al implante, dejados sin contactos dentarios durante la máxima intercuspidación (MIC) y movimientos excéntricos mandibulares. Todas las restauraciones fueron observadas durante un período de 6 meses, evaluando tasa de éxito implantario, complicaciones biológicas y mecánicas. Resultados: Se insertaron 25 implantes en 21 pacientes, obteniéndose un valor promedio de inserción de torque quirúrgico de 45.6 Nt. Durante el período de observación clínico se presentó un 100\% de éxito de sobrevida implantario, no se detectaron complicaciones biológicas, pero sí se presentaron complicaciones mecánicas por fractura de una cúspide vestibular de una restauración provisional acrílica y dos aflojamientos de tornillos. Conclusiones: La temporización unitaria mediante provisorio atornillado es un protocolo predecible y confiable que permite el proceso de oseointegración y la coexistencia en salud de tejido periimplantario y su sistema protésico.
\end{abstract}

Rev. Clin. Periodoncia Implantol. Rehabil. Oral Vol. 4(1); 5-8, 2011.

Palabras clave: Temporización inmediata, complicaciones biológicas y mecánicas.

\section{ABSTRACT}

Purpose: This article is a prospective study of implant success rates and of mechanical and biological complications in the assisted-implant rehabilitation treatment of the posterior maxilla by immediate temporization. Material and Methods: The clinical study observed 21 partially edentulous subjects with lip scar and antagonistic natural teeth in the posterior maxilla for a period of 6 months in which conical threaded internal implant connections were inserted with a surface treatment and timed immediately after being inserted into the jawbone by producing an acrylic resin crown on a temporary titanium abutment screwed directly into the implant and left without tooth contacts during the Maximal Intercuspal (MIC) and mandibular eccentric movements. All restorations were observed over a period of 6 months evaluating implant success rate and biological and mechanical complications. Results: 25 implants were inserted into 21 patients yielding an average surgical insertion torque of $45.6 \mathrm{Nt}$. During the clinical observation there was $100 \%$ success of implant survival. No biological complications were detected, but there were mechanical complications - a buccal cusp fracture of an acrylic provisional restoration and two screws loosening. Conclusions: The unit temporization by temporary screwing is a predictable and reliable protocol that allows osseointegration and the coexistence in the health of peri-implant tissue and its prosthetic system.

Rev. Clin. Periodoncia Implantol. Rehabil. Oral Vol. 4(1); 5-8, 2011.

Key words: Immediate temporization, biological and mechanical complication.

\section{INTRODUCCIÓN}

El protocolo original de Brånemark, basado en un procedimiento quirúrgico de dos etapas, dejando el implante sumergido bajo la encía, establecía un período de cicatrización de 4 a 6 meses para la maxila y mandíbula para conseguir la oseointegración ${ }^{(1-4)}$. Sin embargo, la incomodidad y ansiedad del paciente, asociada al período de espera, obliga a plantear protocolos quirúrgicos-protésicos que privilegien la opción de posicionar una corona inmediatamente sobre un implante instalado ${ }^{(2,3)}$.

Varios estudios se han focalizado en la disminución del período de espera establecido por Brånemark en implantes unitarios en zonas estéticas maxilares, temporizándolos inmediatamente, obteniendo tasas de sobrevida implantarias y protésicas sobre el $90 \%(5,6,7)$.

El resultado teórico de una temporización o estética inmediata radica en la minimización de los micro movimientos intra óseos, logrados al obtener valores de torque de inserción superior a $35 \mathrm{NTw}^{(8-11)}$, gracias a los mejoramientos en el diseño macro y microscópico de los actuales implantes que se encuentran en el mercado, y a la protección de la estructura protésica temporal de una carga oclusal directa, debido a la presencia de piezas dentarias vecinas al espacio desdentado a implantar. Clínicamente la opción de temporizar una pieza inmediatamente es un tratamiento de elección en la actualidad, ya que facilita la estética, mejora las condiciones sicológicas de espera del paciente a su restauración definitiva y facilita el proceso de oseointegración, aumentando el contacto del hueso circundate con el implante ${ }^{(12,13,14)}$.

\section{MATERIAL Y MÉTODO}

Se seleccionó un total de 21 pacientes los que serán observados por un período de 3 años. Se obtuvieron resultados iniciales a los 6 meses, durante la fase de temporización inmediata, período que coincide con la fase de término de los procesos de oseointegración establecidos para la maxila y el inicio de la fase de carga funcional directa con coronas cerámicas terminadas.

Reclutados los pacientes, se les instruyó respecto de las condiciones del estudio, enrrolándose todos aquellos sujetos que cumplieron con los criterios de inclusión, previa firma de su respectivo

1. Especialista en Implantología Buco Máxilo Facial. Docente del Programa de Especialización Profesional en Rehabilitación Oral y del Programa de Especialización Profesional en Implantología Buco Máxilo Facial. Facultad de Odontología, Universidad de Chile. Chile.

2. Profesor Asociado. Departamento de Prótesis. Facultad de Odontología, Universidad de Chile. Chile. Director del Programa de Especialización Profesional en Rehabilitación Oral. Facultad de Odontología, Universidad de Chile. Chile. Director de Prótesis del Programa de Especialización Profesional en Implantología Buco Máxilo Facial. Facultad de Odontología, Universidad de Chile. Chile.

3. Especialista en Implantología Buco Máxilo Facial. Ex - Docente del Programa de Especialización en Implantología Buco Máxilo Facial. Facultad de Odontología, Universidad de Chile. Chile.

4. Especialista en Implantología Buco Máxilo Facial. Ex - Docente del Programa de Especialización en Implantología Buco Máxilo Facial. Facultad de Odontología, Universidad de Chile. Chile.

5. Profesor Asistente. Facultad de Odontología, Universidad de Chile. Chile. Director de Cirugía del Programa de Especialización Profesional en Implantología Buco Máxilo Facial. Facultad de Odontología, Universidad de Chile. Chile.

Correspondencia autor: Yerko Leighton F. yerkoleighton@gmail.com. Trabajo recibido el 28/08/2010. Aprobado para su publicación el $10 / 01 / 2011$. 
consentimiento informado, el que fue visado y autorizado por el Comité de Ética de la Universidad de Chile.

Criterios de Inclusión

1. Mayor de 18 años.

2. No presentar alteraciones sistémicas que contraindiquen una cirugía de implantes.

3. Desdentamiento parcial con antagonista dentario.

4. Presentar un segmento unitario desdentado con hueso cicatrizado independiente del lado de la arcada.

5. Disponibilidad ósea para la inserción de un implante de 3.75 por 10.0 mm, como mínimo.

6. Ausencia de compromiso estético por pérdida de encía adherida.

\section{Criterios de Exclusión}

1. Presencia de enfermedad periodontal.

2. Presencia del hábito de fumar.

3. Presencia de Diabetes descontrolada, sin control médico, con valores de glicemia en ayunas superior a $126 \mathrm{mg} / \mathrm{dl}$ o valores de hemoglobina glicosilada superior a un 9 a 10\% (correspondiente a un valor de glicemia 210 a 240 mg/dl).

Los pacientes fueron examinados y se les solicitó exámenes complementarios como fotografías clínicas, montaje de modelos, radiografías periapicales, panorámica y tomografía convencional hipocicloidal de la zona.

\section{Parámetros Observados}

Los siguientes serán los criterios de éxito observados:

\section{Criterios de Éxito Implantario}

Durante el período de observación clínica se valoró:

- Ausencia de dolor espontáneo y a la percusión longitudinal y oblícua.

a. Nivel óseo acorde con formación de ancho biológico.

b. Ausencia de radiolucidez periimplantaria.

c. Ausencia de movilidad evidente.

d. Sonido mate a la percusión.

\section{Criterios de Éxito Protésico}

Durante el período de observación clínica se valoró:

- Aflojamiento de tornillos protésicos.

- Fractura del elemento protésico de resina acrílica.

\section{Criterios de Éxito Biológico}

Durante el período de observación clínica se valoró:

- Ausencia de signos o síntomas inflamatorios del tejido gingival periimplantar.

- Ausencia de exudados periimplantarios.

\section{Procedimiento Quirúrgico}

Los paciente fueron intervenidos en los pabellones de la Escuela de Graduados de la Facultad de Odontología de la Universidad de Chile, siendo premedicados antes de su cirugía mediante terapia profiláctica antibiótica, amoxicilina 2 grs $1 \mathrm{hr}$ antes de la cirugía y analgésicos antiinflamatorios (meloxicam $7.5 \mathrm{mg}$ cada $12 \mathrm{hrs}$ y paracetamol $500 \mathrm{mg}$ cada $8 \mathrm{hrs}$ por 3 días).

El procedimiento quirúrgico comenzó con la infiltración de anestesia local en la zona a tratar, para luego iniciar la elevación de un colgajo de espesor total mediante una incisión supra crestal y dos descargas vestibulares laterales intracreviculares. Una vez visualizado el sitio a implantar se posiciona la cubeta multifuncional desmontable (Universidad de Chile) $)^{(17)}$, en fase de guía quirúrgica para iniciar el fresado, se verifica con la fresa 2.0 la dirección del fresado, junto con evaluar la consistencia y dureza ósea, para determinar los siguientes pasos de fresas o bien sub-fresar el hueso remanente, con el fin de obtener un torque de inserción superior a $35 \mathrm{Ncm}$. Terminado el fresado se inserta el implante sin irrigación y se verifica el torque de inserción y el cumplimiento de la dirección, utilizando la guía quirúrgica. Para finalizar el procedimiento se sutura con seda negra trenzada mediante puntos simples sin tensión, para permitir el acceso inmediato del pilar temporal de titanio utilizado en la carga protésica.

\section{Procedimiento Protésico}

Terminada la cirugía se procede a preparar un provisorio, utilizando un pilar temporal de titanio, empleando la técnica de cubeta multifuncional desmontable (Universidad de Chile) en fase de guía protésica(17), obteniendo así una corona acrílica libre de contactos oclusales en MIC y movimientos excursivos lista para ser atornillada directa al implante.

Terminada la fase quirúrgica y protésica, los pacientes comenzaron un proceso de observación durante 6 meses $\left(2^{\text {do }}, 4^{\text {to }}\right.$ y $6^{\text {to }}$ mes de observación), lo cual fue consignado en una ficha de recolección de datos, observando: éxito de inserción implantario, complicaciones biológicas y complicaciones mecánicas.

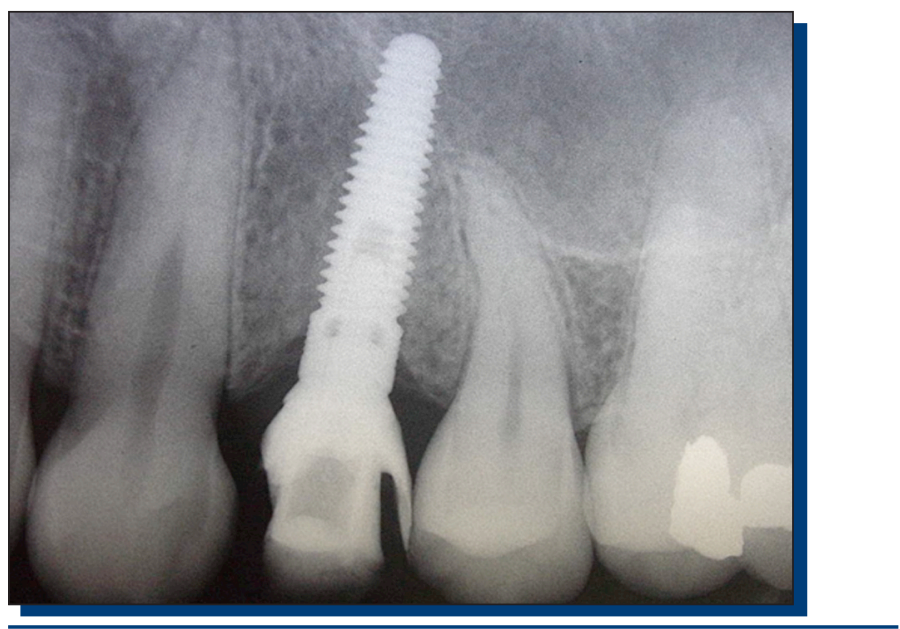

Figura 1. Radiografía periapical de control post quirúrgica.

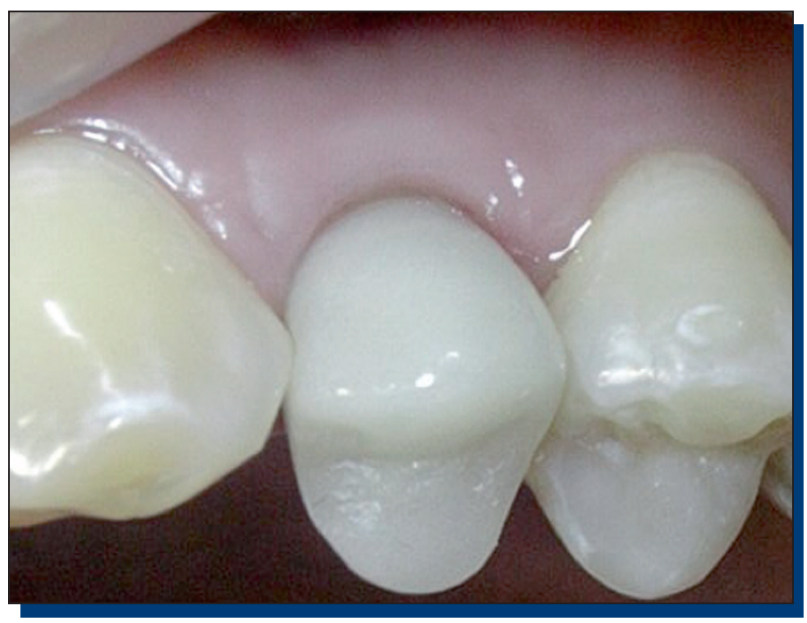

Figura 2. Provisorio acrílico

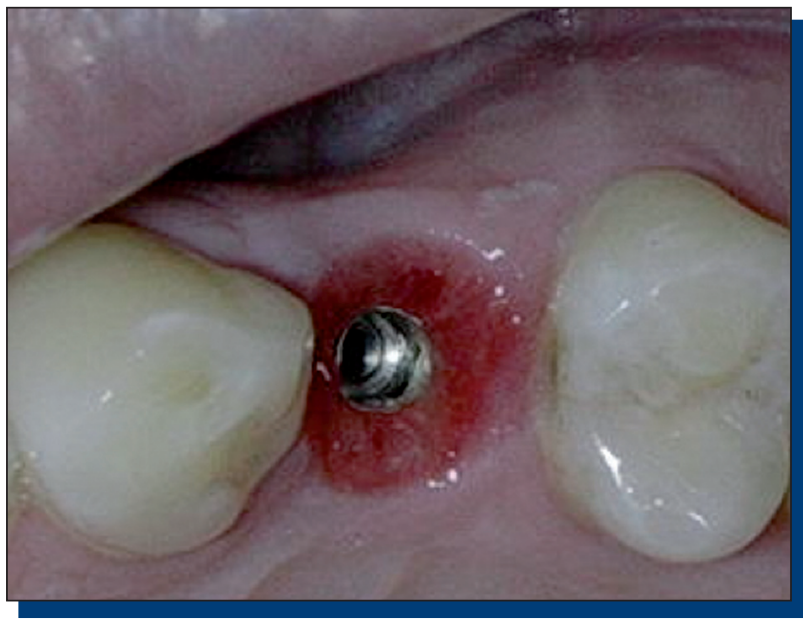

Figura 3. Control tejidos periimplantarios al sexto mes. 


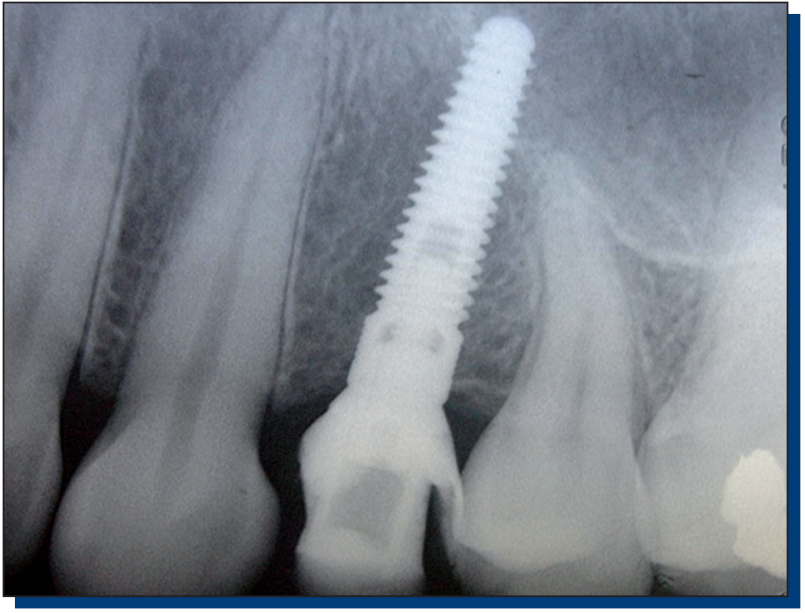

Figura 4. Radiografía periapical de control al sexto mes.

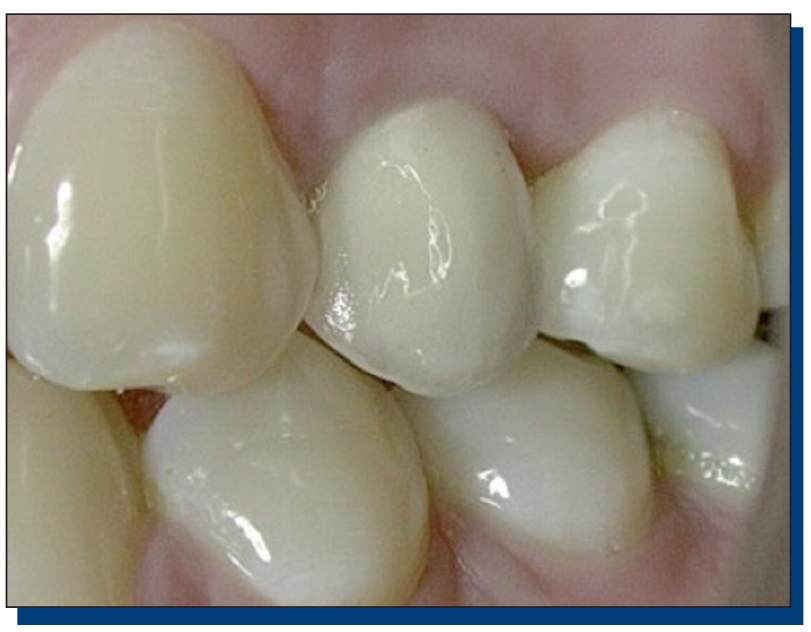

Figura 5. Corona cerámica completa cementada al sexto mes.

\section{RESULTADOS}

Se atendió un total de 21 pacientes instalándose un total de 25 implantes con un torque promedio de inserción quirúrgicos de 45.6 $\mathrm{Ncm}$ sin presentar complicaciones quirúrgicas ni post operatorias, junto con una buena aceptación de los pacientes en relación a su estética inmediata y ausencia de signos inflamatorios post quirúrgicos.

Durante los 6 meses de control se obtuvo un $100 \%$ de éxito de inserción implantario, no se observó presencia de complicaciones biológicas como mucositis o periimplantitis.

El análisis de las complicaciones mecánicas evidenció el aflojamiento de 2 tornillos protésicos, representando un $92 \%$ de éxito, y una fractura de una cúspide protésica de una corona acrílica, representando un $96 \%$ de éxito.

Terminado el proceso de control de 6 meses, el que coincide con la culminación de la fase de oseointegración de la maxila y previo control radiográfico, se procedió a la confección de coronas definitivas libres de metal.

\section{DISCUSIÓN}

El proceso de oseointegración se encuentra muy bien documentado y se ha definido como una íntima y estable unión entre la superficie del titanio y el hueso receptor sometido a cargas funcionales ${ }^{(20)}$. Además, se han definido tiempos de oseointegración de 3 a 6 meses respectivamente en la mandíbula y la maxila, para recibir carga funcional directa.

El mayor conocimiento que se tiene en la actualidad sobre la fisiología ósea y los procesos reparativos de los tejidos duros y blandos,
Tabla 1. Distribución de implantes observados.

\begin{tabular}{|l|c|}
\hline \hline Implante & Número \\
\hline Nobel Biocare Replace Selec Tapered & 4 \\
\hline Biomet 3i Natural Tapered (NT) & 9 \\
\hline Lifecore Renova Tapered & 12 \\
\hline Total & $\mathbf{2 5}$ \\
\hline
\end{tabular}

Tabla 2. Distribución de complicaciones biológicas y mecánicas durante la fase de temporización inmedita.

\begin{tabular}{|l|c|c|c|c|c|}
\hline \hline \multicolumn{3}{|c|}{ Complicaciones Biológicas } & Complicaciones Mecánicas \\
\hline Control & $\begin{array}{c}\text { Éxito } \\
\text { Implantario }\end{array}$ & Mucositis & Periimplantitis & $\begin{array}{c}\text { Fractura } \\
\text { Coronaria }\end{array}$ & $\begin{array}{c}\text { Aflojamiento } \\
\text { de Tomillo }\end{array}$ \\
\hline 2 meses & $100 \%$ & - & - & - & - \\
\hline 4 meses & $100 \%$ & - & - & - & 1 \\
\hline 6 meses & $100 \%$ & - & - & 1 & 1 \\
\hline$\%$ éxito & $100 \%$ & $100 \%$ & $100 \%$ & $96 \%$ & $92 \%$ \\
\hline
\end{tabular}

además del desarrollo tecnológico de los sistemas implantarios en el diseño macroscópico y microscópico ${ }^{(27)}$, han modificado el protocolo quirúrgico y protésico original de Brånemark, cargando y temporizando inmediatamente los implantes, obteniendo resultados similares a las tasas de éxito logradas en los procedimientos de carga convencional(5-7).

La opción de temporización unitaria inmediata es una modalidad de tratamiento exitosa y predecible cuando las condiciones óseas son favorables para la inserción de un implante con un diseño macroscópico adecuado a los requerimientos de estabilidad primaria, y microscópicamente con una superficie tratada que facilite la estabilidad secundaria, en presencia de piezas vecinas que brinden protección de la oclusión directa sobre el implante recién instalado.

La opción de confeccionar un provisorio inmediato sobre un implante recién instalado, versus la opción de sumergir un implante bajo la encía para su cicatrización sin carga funcional, encuentra su explicación en la capacidad de estabilidad ósea progresiva, al ser insertado un implante en un hueso que le permite obtener valores superiores a 32 $\mathrm{Ncm}^{(18)}$ evitando asi la posibilidad de sufrir micro movimientos ${ }^{(9,10)}$ que provoquen pérdida de la intimidad implante hueso y genere procesos de fibro integración.

Evitados los micro movimientos, la estabilidad secundaria comienza sus cambios biológicos, físicos y químicos, lo que permitirá un proceso de oseointegración con un mejor desarrollo de hueso periimplantar como fue reportado por Nkenke y cols.(22), al examinar implantes de carga inmediata en cerdos (Minipigs), evidenciando un alto contacto entre hueso e implante y alta calidad de osteones secundarios junto con pequeños espacios presentes entre ellos en relación al hueso periimplantar.

Las investigaciones de Testori y cols. $(2003)^{(12)}$ plantean tasas de éxito de implantes sobre el $95 \%$ para los protocolos de carga inmediata, al igual que lo expuesto por Romanos y cols. (2005)(22), Andersen $(2002)^{(24)}$, Maló $(2000)^{(26)}$ y Chaushu $(2001)^{(25)}$ para implantes unitarios en maxila.

La influencia de la carga controlada sobre el hueso facilitaría y mejoraría el proceso de oseointegración, Isidor ${ }^{(15)}$, Testori y cols. $(2002)^{(16)}$ y actualmente las investigaciones de Degidi y cols. (2009) $(18,19)$, mostrando un mayor contacto óseo (BIC 76.2\%) en los implantes que recibieron carga oclusal inmediata versus los que siguieron el protocolo tradicional Brånemark sumergido (BIC 62.3\%), lo que influye directamente en el éxito y la tasa de sobrevida implantaria.

La opción de confeccionar un provisorio inmediato, ya sea por técnica directa o bien por impresión y confección de laboratorio, asegura y mejora la evolución de la arquitectura de los tejidos blandos y duros manteniendo una buena coexistencia de salud entre el elemento protésico y su entorno; además de optimizar el proceso de oseointegración, ya que se continúa la regeneración ósea, pero con un estímulo de carga funcional indirecta.

El grado de satisfacción estética de los pacientes es alto, elevando su autoestima y aumentando el compromiso con su tratamiento. 


\section{CONCLUSIONES}

El proceso de temporización inmediata unitaria se desarrolla bajo un protocolo simple, reproducible, predecible, con alto nivel de éxito clínico, de gran aceptación psicológica, obteniendo además una coexistencia en salud del elemento protésico con sus tejidos periimplantarios, sin embargo, se pueden presentar complicaciones mecánicas menores, altamente predecibles producto de la fragilidad de la resinas, lo que no pone en riesgo la rehabilitación final.

\section{REFERENCIAS BIBLIOGRÁFICAS}

1. Brånemark $\mathrm{PI}$, Hansson $\mathrm{BO}$, Adell $\mathrm{R}$ et al. Osseointegrated implants in the treatment of the edentulous jaw. Experience from a 10-year period. Scand J Reconstr Surg, 1977; 16(suppl): 1-132.

2. Vandamme K, Naert J, Geris L, Vander Sloten J. The effect of micromotion on the tissue response around immediatetely loaded roughened titanium implants in the rabbit. Eur J Oral Sci, 2007; 115: 21-29.

3. Duyck J, Vandamme K, Geris L et al. The influence of micromotion on the tissue differentiation around immediately turned titanium implants. Arch Oral Biol, 2006; 51: 1-9.

4. Albrektsson T, Zarb G, Worthington P, Eriksson AR. The long-term efficacy of currently used dental implants: A review and proposed criteria for success. Int J Oral Maxillofac Impl, 1986; 1: 11-25.

5. Rocci A, Martignoni M, Gottlow J, Rangert B. Immediate function of single and partial reconstructions in the maxilla using MK IV fixtures. A retrospective analysis. Appl Osseointegration Res, 2001; 2: 22-26.

6. Wohrle PS. Single-tooth replacement in the aesthetic zone with immediate provisionalization: Fourteen consecutive cases reports. Prac Periodontics Aesthet Dent, 1998; 10: 1107-1114.

7. Hui E, Chow J, Li D, Liu J, Wat P, Law H. Immediate provisional for single-tooth implant replacement with Brånemark System: Preliminary report. Clin Implant Dent Relat Res, 2001; 3: 79-86.

8. Kan JYK, Rungcharassaeng K. Immediate placementand provisionalization of maxillary anterior single implants: A surgical and prosthodontic rationale. Pract Periodontics Aesthet Dent, 2000; 12(9): 817-824.

9. Szmukler-Moncler S, Salama H, Reingewirtz Y, Dubruille JH. Timing of loading and effect of micromotion on bone-dental implant interface: A review of experimental literature. J Biomed Mater Res (Appl Biomater), 1998; 43(2): 192-203.

10. Szmukler-Moncler S, Piattelli A, Favero GA, Dubruille JH. Considerations preliminary to the application of early and immediate loading protocols in dental implantology. Clin Oral Impl Res, 2000; 11(1): 12-25.

11. Kawahara $H$, Kawahara D, Hayakawa $M$ et al. Osseointegration under immediate loading: Biomechanical stress-strain and bone formationresorption. Implant Dent, 2003; 12(1): 61-68.

12. Testori T, Bianchi F, Del Fabbro M, Szmukler-Moncler S, Francetti L, Weinstein RL. Immediate non-occlusal loading vs early loading in partially edentulous patients. Pract Proced Aesthet Dent, 2003; 15(10): 787-794.

13. Gazpski R, Wang HL, Mascarenhas P, Lang NP. Critical review of immediate implant loading. Clin Oral Implants Res, 2003; 14: 515-527.

14. Proussaefs P, Kant J, Lozada J, Kleimman A, Farnos A. A effect of immediate loading with threaded hydroxyapatite-coasted root-formn implants on single premolar replacements: A preliminary report. Int J Oral Maxillofac Implants, 2002; 17: 567-572.

15. Isidor F. Influence of forces on peri-implant bone. Clin Oral Implants Res, 2006; 17(suppl 2): 8-18.
16. Testori T, Szmukler-Moncler S, Francete L, Del Fabbro M, Trissi $P$, Weinstein RL. Healing osseottite implants under sumerged and immediate loading condition in a single patient: A case report and interface analysis after 2 moths. Int J Periodontics Restorative Dent, 2002; 22: 345-353.

17. Leighton Y, Carvajal JC, Wolnitzky A, Silva R, Von Marttens A. Protocolo protésico-quirúrgico en temporización inmediata de dientes unitarios, utilizando una cubeta multifuncional desmontable. Rev Clin Periodoncia Implantol Rehabil Oral, 2010; 3(1): 34-38.

18. Degidi M, Piattelli A, Shibli JA, Perrotti V, lezzi G. Early bone formation around immediately restored implants with and without occlusal contact: A human histologic and histomorphometric evaluation. Case report. Int J Oral Maxillofac Implants, 2009 Jul-Aug; 24(4): 734-739.

19. Degidi M, Piattelli A, Shibli JA, Perrotti V, lezzi G. Bone formation around immediately loaded and submerged dental implants with a modified sandblasted and acid-etched surface after 4 and 8 weeks: A human histologic and histomorphometric analysis. Int J Oral Maxillofac Implants, 2009 Sep-Oct; 24(5): 896-901.

20. Brånemark PI, Breine U, Adell R, Hansson BO, Linström J, Ohlsson A. Intra-osseous anchorage of dental prostheses. I experimental studies. Scand J Plast Reconstr Surg, 1969; 3: 81-100.

21. Lekholm U. Immediate/early loading of oral implants in compromised patients. Periodontology 2000, 2003; 33: 194-203.

22. Nkenke E, Fenner M, Vairaktaris EG, Radespiel-Tröger M. Immediate versus delayed loading of dental implants in the maxillae of minipigs. Part II: Histomorphometric analysis. Int J Oral Maxillofac Implants, 2005; 20: 540-546.

23. Romanos GE, Testori T, Degidi D, Piattelli A. Histological and histomorphometrical findings from retrieved immediately occlusally loaded implants in humans. J Periodontol, 2005; 76: 1823-1832.

24. Andersen E, Haanaes HR, Knutsen BM. Immediate loading of singletooth ITI implants in the anterior maxilla: A prospective 5-years pilot study. Clin Oral Implants Res, 2002; 13: 281-287.

25. Chaushu G, Chaushu S, Tzohar A, Dayan D. Immediate loading of single-tooth implants: Immediate versus non-immediate implantation. A clinical report. Int J Oral Maxillofac Implants, 2001; 16: 267-272.

26. Malo $P$, Rangert B, Dvärsäter L. Immediate function of Brånemark implants in the esthetic zone: A retrospective clinical study with 6 months to 4 years of follow-up. Clin Implant Dent Relat Res, 2000; 3: 138-146.

27. Deporter DA, Watson PA, Pilliar RM et al. A histological assessment of the initial healing response adjacent to porous surfaced Ti alloy dental implants in dogs. J Dent Res, 1986; 65: 1064-1070. 